\title{
Le volume des idéaux d'opérateurs classiques
}

par

\section{JEAN SAINT RAYMOND (Paris)}

Abstract. For $1 \leqslant p \leqslant \infty$, let $B_{n}^{p}$ be the set of operators $t$ on $l_{n}^{2}$ such that the sequence of the $n$ eigenvalues of $|t|$ belongs to the unit ball of $l_{n}^{p}$. We explicitly compute the volume of $B_{n}^{p}$, and give asymptotic evaluation of this volume when $n$ goes to infinity.

On considère ici, pour $1 \leqslant p \leqslant \infty$, la boule unité $B_{n}^{p}$ (resp. $\tilde{B}_{n}^{p}$ ) de l'idéal d'opérateurs réel (resp. complexe) sur $l_{n}^{2}$ définie par $\sum_{j=1}^{n} \lambda_{j}^{p} \leqslant 1$, où les $\lambda_{j}$ sont les $n$ valeurs propres de $|t|=\left(t^{*} t\right)^{1 / 2}$.

On donne une formule explicite pour le volume de $B_{n}^{p}$ et $\widetilde{B}_{n}^{p}$ en fonction de $n$ et $p$, ainsi que des évaluations asymptotiques de ces quantités quand $n$ tend vers l'infini.

On identifiera toujours un opérateur sur $l_{n}^{2}$ avec sa matrice dans la base canonique $\left(e_{1}, \ldots, e_{n}\right)$ de $l_{n}^{2}$, et l'espace de ces matrices avec $\boldsymbol{R}^{\boldsymbol{n}^{2}}$ ou $\boldsymbol{C}^{n^{2}}$, muni du produit scalaire euclidien $(t, s) \mapsto \operatorname{Re}\left(\operatorname{Trace}\left(t^{*} s\right)\right)$. La norme hilbertienne sur $C^{n^{2}}$ est alors la norme de Hilbert-Schmidt sur $\mathscr{L}\left(l_{n}^{2}\right)$. On obtient ainsi que le volume de $B_{n}^{2}$ est $v_{n^{2}}$ et celui de $\widetilde{B}_{n}^{2}$ est $v_{2 n^{2}}$ si on note $v_{m}=\frac{\pi^{m / 2}}{\Gamma(1+m / 2)}$ le volume de la boule unité euclidienne de $\boldsymbol{R}^{m}$.

THÉORÉme 1. Les volumes de $B_{n}^{p}$ et $\tilde{B}_{n}^{p}$ sont donnés par

$$
\begin{aligned}
\operatorname{vol}\left(B_{n}^{p}\right) & =2^{-n}\left(\prod_{k=1}^{n} k v_{k}\right)^{2} \cdot I_{n}^{p}, \\
\operatorname{vol}\left(\tilde{B}_{n}^{p}\right) & =(2 \pi)^{-n}\left(\prod_{k=1}^{n} 2 k v_{2 k}\right)^{2} \cdot \tilde{I}_{n}^{p}, \\
I_{n}^{p} & =\int_{\Omega_{p}} \prod_{1 \leqslant j<k \leqslant n}\left(\sigma_{k}^{2}-\sigma_{j}^{2}\right) d \sigma_{1} \ldots d \sigma_{n}, \\
\tilde{I}_{n}^{p} & =\int_{\Omega_{p}} \prod_{1 \leqslant j<k \leqslant n}\left(\sigma_{k}^{2}-\sigma_{j}^{2}\right)^{2} \sigma_{1} \sigma_{2} \ldots \sigma_{n} d \sigma_{1} \ldots d \sigma_{n},
\end{aligned}
$$

où. $\Omega_{p}=\left\{\left(\sigma_{1}, \ldots, \sigma_{n}\right) \mid 0<\sigma_{1}<\ldots<\sigma_{n}\right.$ et $\left.\sum_{j=1}^{n} \sigma_{j}^{p}<1\right\}$. 
L'ensemble des opérateurs singuliers étant négligeable, presque tout opérateur $t$ se met de façon unique sous la forme

$$
t=u h
$$

où $h=\left(t^{*} t\right)^{1 / 2}$ est hermitien positif et $u \in O(n)$ (resp. $\left.U(n)\right)$ dans le cas réel (resp. complexe). Il existe alors $v \in O(n)$ (resp. $U(n)$ ) tel que

$$
h=v^{*} d v
$$

où $d$ est diagonal de valeurs propres positives $\sigma_{1} \leqslant \sigma_{2} \ldots \leqslant \sigma_{n}$. Pour presque tout opérateur $t$, les $\sigma_{j}$ sont deux à deux distinctes, et $v$ est unique à la multiplication près de ses lignes par des scalaires de module 1.

Notons enfin que $t \in B_{n}^{p}$ (resp. $\left.\tilde{B}_{n}^{p}\right)$ si et seulement si $\sum_{j=1}^{n} \sigma_{j}^{p} \leqslant 1$.

Soit $\Phi$ l'application de $O(n) \times O(n) \times \Omega_{p}$ dans $\mathscr{L}\left(\boldsymbol{R}^{n}\right)$ (resp. $\Psi: U(n) \times$ $\left.\times U(n) \times \Omega_{p} \rightarrow \mathscr{L}\left(C^{n}\right)\right)$ définie par

$$
\Phi(u, v, \sigma)=u \circ \sigma \circ v, \quad \Psi(u, v, \sigma)=u \circ \sigma \circ v
$$

en identifiant $\sigma \in \Omega_{p} \subset \boldsymbol{R}^{n}$ avec l'opérateur diagonal de valeurs propres $\left(\sigma_{1}, \ldots, \sigma_{n}\right)$. On a

$$
\begin{array}{lll}
C_{n}^{p}=\Phi\left(O(n) \times O(n) \times \Omega_{p}\right) \subset B_{n}^{p} & \text { et } & \operatorname{vol}\left(B_{n}^{p} \backslash C_{n}^{p}\right)=0, \\
\tilde{C}_{n}^{p}=\Psi\left(U(n) \times U(n) \times \Omega_{p}\right) \subset \widetilde{B}_{n}^{p} & \text { et } & \operatorname{vol}\left(\widetilde{B}_{n}^{p} \backslash \widetilde{C}_{n}^{p}\right)=0 .
\end{array}
$$

On munit $O(n)$ (resp. $U(n)$ ) de la structure riemannienne induite par son plongement dans l'espace euclidien $\boldsymbol{R}^{n^{2}}$ (resp. $C^{n^{2}}=\boldsymbol{R}^{2 n^{2}}$ ) et de la mesure associée $\mu$. Puisque, pour $u \in O(n)$ (resp. $U(n)$ ), les applications $t \mapsto t u$ et $t \mapsto u t$ sont isométriques pour la norme de Hilbert-Schmidt, $\mu$ est une mesure de Haar. Si on note $J(u, v, \sigma)$ (resp. $\tilde{J}(u, v, \sigma)$ ) le jacobien de $\Phi$ (resp. $\Psi$ ) en $(u, v, \sigma)$, c'est-à-dire lẹ déterminant de l'application $\boldsymbol{R}$-linéaire tangente à $\Phi$ (resp. $\Psi)$ restreinte à la co-image $(\operatorname{Ker} T \Phi)^{\perp \cdot}\left(\right.$ resp. $\left.(\operatorname{Ker} T \Psi)^{\perp}\right)$, on aura, puisque les applications $w \mapsto u \cdot w$ et $w \mapsto w \cdot v$ induisent des isométries de l'espace tangent à $O(n)$ (resp. $U(n)$ ) en 1 sur les espaces tangents à $O(n)$ (resp. $U(n))$ en $u$ et $v$,

$$
|J(u, v, \sigma)|=|J(1,1, \sigma)|, \quad|\tilde{J}(u, v, \sigma)|=|\tilde{J}(1,1, \sigma)|,
$$

qui sont constants sur les fibres $\Phi^{-1}(t)$ et $\Psi^{-1}(t)$. Donc

$$
\begin{aligned}
\int_{O(n) \times O(n) \times \Omega_{p}}|J(u, v, \sigma)| d \mu(u) d \mu(v) d \sigma & =\int_{C_{n}^{p}} \operatorname{vol}\left(\Phi^{-1}(t)\right) d t, \\
\int_{U(n) \times U(n) \times \Omega_{p}}|\tilde{J}(u, v, \sigma)| d \mu(u) d \mu(v) d \sigma & =\int_{\mathcal{C}_{n}^{p}} \operatorname{vol}\left(\Psi^{-1}(t)\right) d t
\end{aligned}
$$

où vol $\Phi^{-1}(t)$ et vol $\Psi^{-1}(t)$ désignent les volumes riemanniens des fibres
$\Phi^{-1}(t)$ et $\Psi^{-1}(t)$. Dans le cas réel on a vu que pour presque tout $t, \Phi^{-1}(t)$ a $2^{n}$ points, d'où vol $\left(\Phi^{-1}(t)\right)=2^{n}$.

Dans le cas complexe, $\Psi^{-1}(t)$ est paramétré par

$$
\varrho: \omega \in[0,2 \pi]^{n} \mapsto\left(u \cdot m_{\omega} \cdot v^{*}, v \cdot m_{\omega}^{*}, \sigma\right)
$$

où $m_{\omega}$ est l'opérateur diagonal unitaire de valeurs propres $\left(e^{i \omega_{1}}, \ldots, e^{i \omega_{n}}\right)$. Comme l'application linéaire $T_{e}$ tangente à $\varrho$ multiplie les distances par $\sqrt{2}$, on obtient

On en déduit

$$
\text { vol } \Psi^{-1}(t)=(2 \pi)^{n} \cdot(\sqrt{2})^{n}
$$

$$
\begin{gathered}
\text { vol } B_{n}^{p}=\operatorname{vol} C_{n}^{p}=\frac{1}{2^{n}} \int_{\Omega_{p}}|J(1,1, \sigma)| d \sigma \times(\operatorname{vol} O(n))^{2}, \\
\text { vol } \tilde{B}_{n}^{p}=\operatorname{vol} \tilde{C}_{n}^{p}=\frac{1}{(2 \pi \sqrt{2})^{n}} \int_{\Omega_{p}}|\widetilde{J}(1,1, \sigma)| d \sigma \times(\operatorname{vol} U(n))^{2} .
\end{gathered}
$$

L'espace tangent $V$ à $O(n)$ en 1 est le sous-espace de $\mathscr{L}\left(\boldsymbol{R}^{n}\right)$ formé des opérateurs antisymétriques dont une base orthonormée est

$$
\varepsilon_{j, k}=\frac{e_{j} \otimes e_{k}-e_{k} \otimes e_{j}}{\sqrt{2}}, \quad 1 \leqslant j<k \leqslant n
$$

où $\left(e_{j}\right)$ est la base canonique de $l_{n}^{2}$ et $e_{j} \otimes e_{k}$ identifié à l'opérateur

$$
x \mapsto\left\langle x, e_{k}\right\rangle \cdot e_{j} \text {. }
$$

L'application linéaire tangente à $\Phi$ en $(1,1, \sigma)$ transforme le vecteur tangent $(a, b, x) \in V \times V \times \boldsymbol{R}^{n}$ en

$$
a \sigma+\sigma b+x
$$

Notant alors $\varepsilon_{j, k}^{1}$ et $\varepsilon_{j, k}^{2}$ les vecteurs correspondant à $\varepsilon_{j, k}$ dans le premier et le second facteur $V$, on a

Donc

$$
\begin{aligned}
T \Phi \cdot \varepsilon_{j, k}^{1} & =\frac{\sigma_{k} e_{j} \otimes e_{k}-\sigma_{j} e_{k} \otimes e_{j}}{\sqrt{2}}, \\
T \Phi \cdot \varepsilon_{j, k}^{2} & =\frac{\sigma_{j} e_{j} \otimes e_{k}-\sigma_{k} e_{k} \otimes e_{j}}{\sqrt{2}}, \\
T \Phi \cdot e_{l} & =e_{l} \otimes e_{l} .
\end{aligned}
$$

$$
\left(T \Phi \cdot \varepsilon_{j, k}^{1}\right) \wedge\left(T \Phi \cdot \varepsilon_{j, k}^{2}\right)=\frac{\sigma_{k}^{2}-\sigma_{J}^{2}}{2}\left(e_{j} \otimes e_{k}\right) \wedge\left(e_{k} \otimes e_{j}\right)
$$


et

$$
\begin{aligned}
\left|\left[_{1 \leqslant j<k \leqslant n}\left(T \Phi \cdot \varepsilon_{j, k}^{1}\right) \wedge\left(T \Phi \cdot \varepsilon_{j, k}^{2}\right)\right] \wedge\left[\bigwedge_{1 \leqslant j \leqslant n}\left(T \Phi \cdot e_{j}\right)\right]\right| \\
\quad=\left[\prod_{1 \leqslant j<k \leqslant n}\left|\sigma_{k}^{2}-\sigma_{j}^{2}\right|\right] \times 2^{\cdots n(n-1) / 2}=|J(1,1, \sigma)|,
\end{aligned}
$$

d'où

$$
\operatorname{vol} B_{n}^{p}=2^{-n} \cdot 2^{-n(n-1) / 2}(\operatorname{vol} O(n))^{2} \int_{S_{p}} \prod_{1 \leqslant j<k \leqslant n}\left(\sigma_{k}^{2}-\sigma_{j}^{2}\right) d \sigma
$$

De même l'espace tangent $\widetilde{V}$ à $\mathrm{SU}(n)$ en 1 est le sous-espace de $\mathscr{L}\left(C^{n}\right)$ formé des opérateurs anti-hermitiens dont une base orthonormée est

$$
\begin{array}{rlrl}
\varepsilon_{j, k}=\frac{e_{j} \otimes e_{k}-e_{k} \otimes e_{j}}{\sqrt{2}}, & 1 \leqslant j<k \leqslant n, \\
\eta_{j, k}=i \frac{e_{j} \otimes e_{k}+e_{k} \otimes e_{j}}{\sqrt{2}}, & & 1 \leqslant j<k \leqslant n, \\
\eta_{j}=i e_{j} \otimes e_{j}, & 1 \leqslant j \leqslant n .
\end{array}
$$

L'application tangente à $\Psi$ en $(1,1, \sigma)$ transforme $(a, b, x) \in \tilde{V} \times \tilde{V} \times \boldsymbol{R}^{n}$ en $a \sigma+\sigma b+x$. Il en résulte qu'une base orthonormée de la coimage de $T \Psi$ est formée de

$$
\begin{array}{ll}
\varepsilon_{j, k}^{1} ; \varepsilon_{j, k}^{2} ; \eta_{j, k}^{1} ; \eta_{j, k}^{2}, & 1 \leqslant j<k \leqslant n, \\
\frac{1}{\sqrt{2}}\left(\eta_{j}^{1}+\eta_{j}^{2}\right) ; e_{j}, & 1 \leqslant j \leqslant n,
\end{array}
$$

et que

$$
\begin{aligned}
& T \Psi \cdot \varepsilon_{j, k}^{1}=\frac{\sigma_{k} e_{j} \otimes e_{k}-\sigma_{j} e_{k} \otimes e_{j}}{\sqrt{2}}, \\
& T \Psi \cdot \varepsilon_{j, k}^{2}=\frac{\sigma_{j} e_{j} \otimes e_{k}-\dot{\sigma}_{k} e_{k} \otimes e_{j}}{\sqrt{2}}, \\
& T \Psi \cdot \eta_{j, k}^{1}=\frac{\sigma_{k} \cdot i e_{j} \otimes e_{k}+\sigma_{j} \cdot i e_{k} \otimes e_{j}}{\sqrt{2}}, \\
& T \Psi \cdot \eta_{j, k}^{2}=\frac{\sigma_{j} i e_{j} \otimes e_{k}+\sigma_{k} i e_{k} \otimes e_{j}}{\sqrt{2}}, \\
& T \Psi \cdot \frac{1}{\sqrt{2}}\left(\eta_{j}^{1}+\eta_{j}^{2}\right)=\sigma_{j} \sqrt{2} i e_{j} \otimes e_{j}, \\
& T \Psi \cdot e_{j}=e_{j} \otimes e_{j} .
\end{aligned}
$$

\section{Donc}

$$
\begin{aligned}
|\tilde{J}(1,1, \sigma)| & =\mid\left[\bigwedge_{1 \leqslant j \leqslant k \leqslant n}\left(T \Psi \cdot \varepsilon_{j, k}^{1}\right) \wedge\left(T \Psi \cdot \varepsilon_{j, k}^{2}\right) \wedge\left(T \Psi \cdot \eta_{j, k}^{1}\right) \wedge\left(T \Psi \cdot \eta_{j, k}^{2}\right)\right] \wedge \\
\wedge\left[\bigwedge_{1 \leqslant j \leqslant n}\left(T \Psi \cdot e_{j}\right) \wedge\left(T \Psi \cdot\left(\frac{1}{\sqrt{2}}\left(\eta_{j}^{1}+\eta_{j}^{2}\right)\right)\right)\right] \mid & \\
& =\prod_{1 \leqslant j<k \leqslant n} \frac{\left(\sigma_{k}^{2}-\sigma_{j}^{2}\right)^{2}}{4} \times \prod_{j=1}^{n} \sigma_{j} \cdot \sqrt{2} \\
& =2^{\cdots \cdots n(n-1)} \cdot 2^{n / 2} \prod_{j=1}^{n} \sigma_{j} \prod_{1 \leqslant j<k \leqslant n}\left(\sigma_{k}^{2}-\sigma_{j}^{2}\right)^{2}
\end{aligned}
$$

d'où

$\operatorname{vol} \widetilde{B}_{n}^{p}=(2 \pi \sqrt{2})^{-n} \cdot 2^{-n(n-1)} \cdot 2^{n / 2} \cdot[\operatorname{vol} U(n)]^{2} \int_{\Omega_{p}} \prod_{1 \leqslant j<k \leqslant n}\left(\sigma_{k}^{2}-\sigma_{j}^{2}\right)^{2} \prod_{j=1}^{n} \sigma_{j} d \sigma$.

En considérant l'application $u \mapsto u\left(e_{1}\right)$ de $O(n)$ sur $S^{n-1}$ (resp. $U(n)$ sur $S^{2 n-1}$ ) dont la fibre est isométrique à $O(n-1)$ (reșp. $U(n-1)$ ), on obtient comme plus haut, en examinant l'application tangente,

$$
\operatorname{vol}(O(n))=2^{(n-1) / 2} \operatorname{vol}\left(S^{n-1}\right) \cdot \operatorname{vol}(O(n-1))
$$

et puisque $O(1)=\{+1,-1\}$

$$
\begin{aligned}
\operatorname{vol}(O(n)) & =2^{n(n-1) / 4} \times \prod_{k=1}^{n-1} \operatorname{vol}\left(S^{k}\right) \times 2 \\
& =2^{1+n(n-1) / 4} \times \prod_{k=2}^{n} k v_{k}=2^{n(n-1) / 4} \prod_{k=1}^{n} k v_{k},
\end{aligned}
$$

donc

$$
\operatorname{vol}\left(B_{n}^{p}\right)=2^{-n} \cdot\left(\prod_{k=1}^{n} k v_{k}\right)^{2} \int_{\Omega_{p}} \prod_{1 \leqslant j<k \leqslant n}\left(\sigma_{k}^{2}-\sigma_{j}^{2}\right) d \sigma_{1} \ldots d \sigma_{n},
$$

qui est la formule cherchée. De même,

$$
\operatorname{vol}(U(n))=2^{n-1} \cdot \operatorname{vol}\left(S^{2 n-1}\right) \cdot \operatorname{vol}(U(n-1))
$$

et puisque $U(1)=\left\{e^{i \theta} \mid 0 \leqslant \theta \leqslant 2 \pi\right\}$,

$$
\operatorname{vol}(U(n))=2 \pi \cdot 2^{n(n-1) / 2} \cdot \prod_{k=2}^{n} \operatorname{vol}\left(S^{2 k-1}\right)=2^{n(n-1) / 2} \cdot \prod_{k=1}^{n}\left(2 k v_{2 k}\right),
$$

donc

$$
\operatorname{vol}\left(\tilde{B}_{n}^{p}\right)=(2 \pi)^{-n} \cdot\left(\prod_{k=1}^{n} 2 k v_{2 k}\right)^{2} \cdot \int_{\Omega_{p}} \prod_{1 \leqslant j<k \leqslant n}\left(\sigma_{k}^{2}-\sigma_{j}^{2}\right)^{2} \prod_{j=1}^{n} \sigma_{j} d \sigma
$$

ce qui achève la démonstration. 
THÉORÈME 2. Si on pose

$$
P_{n}=\prod_{k=1}^{n}\left(k v_{k}\right), \quad \tilde{P}_{n}=\prod_{k=1}^{n}\left(2 k v_{2 k}\right)
$$

on $a$, pour $n$ tendant vers l'infini

$$
\begin{aligned}
\log P_{n}^{1 / n^{2}} & =\frac{1}{4} \log \left(2 \pi e^{3 / 2}\right)-(\log n) / 4+O((\log n) / n), \\
\log \tilde{P}_{n}^{1 / 2 n^{2}} & =\frac{1}{4} \log \left(2 \pi e^{3 / 2}\right)-(\log 2 n) / 4+O((\log n) / n) .
\end{aligned}
$$

Ceci se déduit aisément de $v_{k}=\frac{\pi^{k / 2}}{\Gamma(1+k / 2)}$ et de la formule de Stirling.

THÉORÈme 3. La limite, quand $n$ tend vers l'infini, de $\left(I_{n}^{\left(x_{i}\right.}\right)^{1 / n^{2}}$ vaut $1 / 2$, ainsi que celle de $\left(\tilde{I}_{n}^{\infty}\right)^{1 / 2 n^{2}}$.

Ces deux résultats se prouvent de manière similaire. Nous laissons au lecteur la démonstration pour $\left(\tilde{I}_{n}^{(\alpha)}\right)^{1 / 2 n^{2}}$.

Soit $\delta_{k}$ le diamètre d'ordre $k$ de $[0,1]$, défini par

$$
\delta_{k}=\sup _{\tau_{1}, \ldots, \tau_{k} \in[0,1]}\left(\prod_{1 \leqslant i<j \leqslant k}\left|\tau_{j}-\tau_{i}\right|\right)^{2 / k(k-1)} .
$$

On sait ([2], p. 268) que $\delta_{k}$ tend en décroissant vers le diamètre transfini de $[0,1]$ qui vaut $1 / 4$. Il en résulte que

$$
I_{n}^{\infty} \leqslant \int_{\Omega_{x_{1}}}\left(\delta_{n}\right)^{n(n-1) / 2} d \sigma_{1} \ldots d \sigma_{n}=\left(\delta_{n}\right)^{m(n-1) / 2} \operatorname{vol}\left(\Omega_{x_{1}}\right)=\left(\delta_{n}\right)^{m(n-1) / 2} \times(1 / n !),
$$

donc

$$
\limsup _{n \rightarrow \infty}\left(I_{n}\right)^{1 / n^{2}} \leqslant \limsup _{n \rightarrow \infty}\left(\delta_{n}\right)^{1 / 2}=\sqrt{1 / 4}=1 / 2
$$

On sait aussi que $\delta_{k}$ est approché par la suite

En effet

$$
\tau_{j}=\sin ^{2} \frac{(j-1) \pi}{2(k-1)}
$$

Alors si on pose

$$
\prod_{1 \leqslant i<j \leqslant k}\left(\sin ^{2} \frac{(j-1) \pi}{2(k-1)}-\sin ^{2} \frac{(i-1) \pi}{2(k-1)}\right)=\left(\frac{2(k-1)}{4^{k-1}}\right)^{k / 2}
$$

$$
\begin{gathered}
\varphi(\sigma)=\prod_{1 \leqslant i<j \leqslant n}\left(\sigma_{j}^{2}-\sigma_{i}^{2}\right), \\
\sigma^{*}=\left(\sigma_{1}^{*}, \ldots, \sigma_{n}^{*}\right) \quad \text { où } \quad \sigma_{j}^{*}=\sin \frac{(j-1) \pi}{2(n-1)},
\end{gathered}
$$

on aura $\varphi(\sigma) \geqslant \varphi\left(\sigma^{*}\right)(1-\varepsilon)^{n^{2}}$ sur un voisinage de $\sigma^{*}$ de mesure suffisamment grande pour que

$$
\left(\int_{\Omega_{\infty}} \varphi(\sigma) d \sigma\right)^{1 / n^{2}} \geqslant\left(\varphi\left(\sigma^{*}\right)\right)^{1 / n^{2}}(1-2 \varepsilon)
$$

pour $n$ assez grand. De façon plus précise, si

et

$$
m=\inf _{i \neq j}\left|\sigma_{i}^{* 2}-\sigma_{f}^{* 2}\right|=\sin ^{2} \frac{\pi}{2(n-1)} \geqslant \frac{1}{(n-1)^{2}}
$$

on a

$$
\sup _{i}\left|\sigma_{i}-\sigma_{i}^{*}\right| \leqslant \delta m
$$

$$
\left|\sigma_{i}^{2}-\sigma_{i}^{* 2}\right|=\left(\sigma_{i}+\sigma_{i}^{*}\right)\left|\sigma_{i}-\sigma_{i}^{*}\right| \leqslant 2 \delta m,
$$

d'où

$$
\begin{aligned}
\sigma_{j}^{2}-\sigma_{i}^{2} & \geqslant \sigma_{j}^{* 2}-\sigma_{i}^{* 2}-\left|\sigma_{j}^{2}-\sigma_{j}^{* 2}\right|-\left|\sigma_{i}^{2}-\sigma_{i}^{* 2}\right| \\
& \geqslant \sigma_{j}^{* 2}-\sigma_{i}^{* 2}-4 \delta m \geqslant(1-4 \delta)\left(\sigma_{j}^{* 2}-\sigma_{i}^{* 2}\right)
\end{aligned}
$$

et

$$
\varphi(\sigma) \geqslant(1-4 \delta)^{n(n-1) / 2} \varphi\left(\sigma^{*}\right) .
$$

Cette condition est réalisée sur l'ensemble

$$
[0, \delta m] \times \prod_{2}^{n-1}\left[\sigma_{i}^{*}-\delta m, \sigma_{i}^{*}+\delta m\right] \times[1-\delta m, 1]
$$

dont la mesure est $2^{n-2}(\delta m)^{n}$, donc

et avec $\delta=1 / 2(n+1)$

$$
I_{n}^{\alpha_{1}} \geqslant(1-4 \delta)^{n(n-1) / 2} \cdot 2^{n-2}(\delta m)^{n} \varphi\left(\sigma^{*}\right)
$$

$$
\left(I_{n}^{\infty}\right)^{1 / n^{2}} \geqslant 2^{-2 / n^{2}} \frac{(n-1)^{(n-5) / 2 n}}{(n+1)^{(n+1) / 2 n}}\left(\varphi\left(\sigma^{*}\right)\right)^{1 / n^{2}} ;
$$

et puisque $\left(\varphi\left(\sigma^{*}\right)\right)^{1 / n^{2}}=\left(\frac{2(n-1)}{4^{n-1}}\right)^{1 / 2 n}$,

$$
\liminf _{n \rightarrow \infty}\left(I_{n}^{\infty}\right)^{1 / n^{2}} \geqslant 1 / 2,
$$

ce qui achève la démonstration.

Corollaire 4. Lorsque $n$ tend vers l'infini, on a

$$
\left[\operatorname{vol}\left(B_{n}^{(\alpha)}\right)\right]^{1 / n^{2}} \sim \frac{1}{2} \sqrt{\frac{2 \pi e^{3 / 2}}{n}}, \quad\left[\operatorname{vol}\left(\widetilde{B}_{n}^{(\alpha)}\right)\right]^{1 / 2 n^{2}} \sim \frac{1}{2} \sqrt{\frac{\pi e^{3 / 2}}{n}} .
$$

Ceci résulte des trois théorèmes précédents, et améliore un résultat de Gluskin prouvant l'existence d'un $C>0$ tel que, pour tout $n,\left(\operatorname{vol}\left(B_{n}^{*}\right)\right)^{1 / n^{2}}$ $\geqslant \frac{C}{\sqrt{n}}$. 
On va généraliser le résultat précédent pour évaluer $\left(I_{n}^{p}\right)^{1 / n^{2}}$. Posons, pour $0<q<+\infty$,

$$
\Delta_{n}(q)=\sup _{0 \leqslant \tau_{1} \leqslant \tau_{2} \leqslant \ldots \leqslant \tau_{n}} n^{1 / q} \cdot \frac{\left(\prod_{1 \leqslant i<j \leqslant n}\left|\tau_{j}-\tau_{i}\right|\right)^{2 / n\left(n+n^{\prime \prime}-1\right)}}{\left(\sum \tau^{q}\right)^{1 / q}},
$$

qui tend vers le diamètre d'ordre $n$ de $[0,1]$ quand $q$ tend vers l'infini.

Lemme 5. La suite $\Delta_{n}(q)$ décroit vers une quantité $\Delta(q)$ quand $n$ tend vers l'infini et

$$
\Delta(q) \geqslant \frac{1}{4}\left(\frac{\sqrt{\pi} \cdot \Gamma(q+1)}{\Gamma(q+1 / 2)}\right)^{1 / 4} .
$$

Pour $q \geqslant 1 / 2$ on a $1 / 4 \leqslant \Delta(q) \leqslant 4$.

Soient $\tau_{1} \leqslant \tau_{2} \leqslant \ldots \leqslant \tau_{n+1}$ des nombres positifs. Alors, si $n \geqslant 2$,

$$
\begin{aligned}
\left(\prod_{1 \leqslant i<j \leqslant n+1}\left|\tau_{j}-\tau_{i}\right|\right)^{n-1} & =\prod_{k=1}^{n+1}\left(\prod_{\substack{1, i<j \leqslant n \\
i, j \neq k}}\left|\tau_{j}-\tau_{i}\right|\right) \\
& \leqslant \prod_{k=1}^{n+1}\left[n^{-1 / q} \Delta_{n}(q)\left(\sum_{i \neq k} \tau^{q}\right)^{1 / q}\right]^{n(n-1) / 2} \\
& \leqslant\left(n^{-1 / q} \Delta_{n}(q)\right)^{n(n+1)(n-1) / 2}\left[\prod_{k=1}^{n+1}\left(\sum_{i \neq k} \tau^{q}\right)\right]^{(n-1) / 2 q}
\end{aligned}
$$

En vertu de l'inégalité entre moyennes arithmétique et géométrique, ceci se majore par

$$
\begin{aligned}
\left(n^{-1 / q} \Delta_{n}(q)\right)^{(n+1)(n-1) / 2}\left[\frac{1}{n+1} \sum_{k=1}^{n+1}\left(\sum_{i \neq k} \tau_{i}^{q}\right)\right]^{n(n+1)(n-1) / 2 q} \\
=\left[(n+1)^{-1 / q} \Delta_{n}(q)\left(\sum_{i=1}^{n+1} \tau_{i}^{q}\right)\right]^{(1 / q)(n(n+1)(n-1) / 2)}
\end{aligned}
$$

d'où $\Delta_{n+1}(q) \leqslant \Delta_{n}(q) \leqslant \ldots \leqslant \Delta_{2}(q)=2^{1 / q}$, quantité majorée par 4 si $q \geqslant 1 / 2$. Ceci prouve que $\Delta_{n}(q)$ décroit vers $\Delta(q)=\inf _{n} \Delta_{n}(q)$. Si on prend

$$
\tau_{j}=\sin ^{2} \frac{(j-1) \pi}{2(n-1)}, \quad 1 \leqslant j \leqslant n
$$

on a

$$
\Delta_{n}(q) \geqslant \frac{\left(\frac{2(n-1)}{4^{n-1}}\right)^{\frac{n}{2} \cdot \frac{2}{n(n-1)}}}{\left(\frac{1}{n} \sum_{j=0}^{n-1} \sin ^{2 q} \frac{j \pi}{2(n-1)}\right)^{1 / q}}=\frac{[2(n-1)]^{1 /(n-1)}}{4\left(\frac{1}{n} \sum_{j=0}^{n-1} \sin ^{2 q} \frac{j \pi}{2(n-1)}\right)^{1 / 4}}
$$

et puisque

$$
\frac{1}{n} \sum_{j=0}^{n-1} \sin ^{2 q} \frac{j \pi}{2(n-1)} \rightarrow \frac{2}{\pi} \int_{0}^{\pi / 2} \sin ^{2 q} \cdot t \cdot d t=\frac{2}{\pi} \frac{\Gamma(1 / 2) \Gamma(q+1 / 2)}{\Gamma(q+1)}
$$

on obtient

$$
\liminf _{n \rightarrow \infty} \Delta_{n}(q) \geqslant \frac{1}{4}\left(\frac{\sqrt{\pi} \cdot \Gamma(q+1)}{\Gamma(q+1 / 2)}\right)^{1 / 4} .
$$

De plus, si on considère le développement en série de

$$
\frac{d^{2}}{d q^{2}}(\log \Gamma(q+1)-\log \Gamma(q+1 / 2))
$$

on voit que $\log \frac{\Gamma(q+1) \cdot \sqrt{\pi}}{\Gamma(q+1 / 2)}$ est fonction concave de $q$, nulle en 0 . Il en résulte que $\frac{1}{q} \log \frac{\sqrt{\pi} \cdot \Gamma(q+1)}{\Gamma(q+1 / 2)}$ est décroissante de $q$, donc positive puisque cette fonction tend vers 0 à l'infini. Ceci achève la démonstration du lemme.

Lemme 6. Il existe un $\tau^{*}=\left(\tau_{1}^{*}, \ldots, \tau_{n}^{*}\right)$ tel que

$$
\begin{gathered}
\sum_{i=1}^{n} \tau_{i}^{* q}=1, \\
\prod_{1 \leqslant i<j \leqslant n}\left|\tau_{j}^{*}-\tau_{i}^{*}\right|=\left(\frac{\Delta_{n}(q)}{n^{1 / q}}\right)^{n(n-1) / 2}, \quad m=\inf _{i \neq j}\left|\tau_{j}^{*}-\tau_{i}^{*}\right| \geqslant\left(\frac{2}{n(n-1)}\right)^{1+1 / q} .
\end{gathered}
$$

Par homogénéité, $\Delta_{n}(q)$ peut être défini comme la borne supérieure de $n^{1 / q} \psi(\tau)$ sur l'ensemble des $\tau$ vérifiant $0 \leqslant \tau_{1} \leqslant \tau_{2} \leqslant \ldots \leqslant \tau_{n}$ et $\|\tau\|_{q}=1$ si

$$
\psi(\tau)=\prod_{1 \leqslant i<j \leqslant n}\left|\tau_{j}-\tau_{i}\right|
$$

borne qui est atteinte par compacité, en un $\tau^{*}$. Il est clair que $\tau_{1}^{*}$ est nul, car $\psi\left(0, \tau_{2}^{*}-\tau_{1}^{*}, \ldots, \tau_{n}^{*}-\tau_{1}^{*}\right)=\psi\left(\tau^{*}\right)$ et

$$
\left\|0, \tau_{2}^{*}-\tau_{1}^{*}, \ldots, \tau_{n}^{*}-\tau_{1}^{*}\right\|_{q}<\left\|\tau^{*}\right\|_{q} \quad \text { si } \quad \tau_{1}^{*} \neq 0 .
$$

On a donc, puisque $\log \psi$ est maximum en $\tau^{*}$ sur $\left\{\|\tau\|_{q}=1\right\}$,

$$
\sum_{j=2}^{n} \frac{d \tau_{j}}{\tau_{j}^{*}}+\sum_{2 \leqslant i<j \leqslant n} \frac{d \tau_{j}-d \tau_{i}}{\tau_{j}^{*}-\tau_{i}^{*}}=\lambda \sum_{i=2}^{n}\left(\tau_{i}^{*}\right)^{q-1} d \tau_{i}
$$

et donc

$$
\alpha_{i}=\sum_{j \neq i} \frac{1}{\tau_{i}^{*}-\tau_{j}^{*}}=\lambda\left(\tau_{i}^{*}\right)^{q-1}, \quad i=2,3, \ldots, n .
$$


Il en résulte que

$$
\sum_{i=1}^{n} \tau_{i}^{*} \alpha_{i}=\sum_{i=2}^{n} \tau_{i}^{*} \alpha_{i}=\sum_{1 \leqslant i<j \leqslant n} \frac{\tau_{i}^{*}-\tau_{j}^{*}}{\tau_{i}^{*}-\tau_{j}^{*}}=\sum_{i=1}^{n} \lambda\left(\tau_{i}^{*}\right)^{q}=\lambda=n(n-1) / 2 .
$$

De plus, pour $2 \leqslant k \leqslant n$

$$
\begin{aligned}
\sum_{i=k}^{n} \alpha_{i} & =\lambda \sum_{i=k}^{n}\left(\tau_{i}^{*}\right)^{q-1} \leqslant \frac{n(n-1)}{2} \sum_{i=2}^{n}\left(\tau_{i}^{*}\right)^{q-1} \\
& =\sum_{i=k}^{n}\left(\sum_{i \neq i} \frac{1}{\tau_{i}^{*}-\tau_{j}^{*}}\right)=\sum_{j<k \leqslant i} \frac{1}{\tau_{i}^{*}-\tau_{j}^{*}} \geqslant \sum_{i=k}^{n} \frac{1}{\tau_{i}^{*}-\tau_{k-1}^{*}} ;
\end{aligned}
$$

ceci donne, pour $k=2$,

$$
\begin{aligned}
\sum_{i=2}^{n} \frac{1}{\tau_{i}^{*}} \leqslant \frac{n(n-1)}{2} \sum_{i=2}^{n}\left(\tau_{i}^{*}\right)^{q-1} & \leqslant \frac{n(n-1)}{2} \sum_{i=2}^{n}\left(\tau_{i}^{*}\right)^{q \cdot q /(q+1)}\left(\frac{1}{\tau_{i}^{*}}\right)^{1 /(q+1)} \\
& \leqslant \frac{n(n-1)}{2}\left(\sum_{i=2}^{n}\left(\tau_{i}^{*}\right)^{q}\right)^{q /(q+1)}\left(\sum_{i=2}^{n} \frac{1}{\tau_{i}^{*}}\right)^{1 /(q+1)} \\
& \leqslant \frac{n(n-1)}{2}\left(\sum_{i=2}^{n} \frac{1}{\tau_{i}^{*}}\right)^{1 /(q+1)}
\end{aligned}
$$

donc

$$
\sum_{i=2}^{n} \frac{1}{\tau_{i}^{*}} \leqslant\left(\frac{n(n-1)}{2}\right)^{(q+1) / q}
$$

et

$$
\begin{aligned}
\sum_{i=2}^{n}\left(\tau_{i}^{*}\right)^{q-1} & \leqslant \sum_{i=2}^{n}\left(\tau_{i}^{*}\right)^{q \cdot q(q+1)}\left(\frac{1}{\tau_{i}^{*}}\right)^{1 /(q+1)} \leqslant\left(\sum_{i=2}^{n}\left(\tau_{i}^{*}\right)^{q}\right)^{q /(q+1)}\left(\sum_{i=2}^{n} \frac{1}{\tau_{i}^{*}}\right)^{1 /(q+1)} \\
& \leqslant\left(\sum_{i=2}^{n} \frac{1}{\tau_{i}^{*}}\right)^{1 /(q+1)} \leqslant\left(\frac{n(n-1)}{2}\right)^{1 / q}
\end{aligned}
$$

c'est-à-dire enfin

$$
\frac{1}{\tau_{k}^{*}-\tau_{k-1}^{*}} \leqslant \sum_{i=k}^{n} \frac{1}{\tau_{i}^{*}-\tau_{k-1}^{*}} \leqslant \frac{n(n-1)}{2} \sum_{i=2}^{n}\left(\tau_{i}^{*}\right)^{4-1} \leqslant\left(\frac{n(n-1)}{2}\right)^{1+1 / 4},
$$

d'où le lemme puisque

$$
m=\inf _{k}\left(\tau_{k}^{*}-\tau_{k-1}^{*}\right) \geqslant\left(\frac{2}{n(n-1)}\right)^{1+1 / 4} \geqslant\left(\frac{1}{n-1}\right)^{2+2 / 4} .
$$

Théorème 7. Lorsque $n$ tend vers l'infini,

$$
\begin{aligned}
&\left(I_{n}^{p}\right)^{1 / n^{2}} \sim n^{-1 / p} \sqrt{\Delta(p / 2)}, \\
&\left(\tilde{I}_{n}^{p}\right)^{1 / 2 n^{2}} \sim n^{-1 / p} \sqrt{\Delta(p / 2)} .
\end{aligned}
$$

On a comme dans le théorème 3 , pour tout $\sigma \in \Omega_{p} \subset \Omega_{\text {x }}$

$$
\varphi(\sigma) \leqslant\left[\frac{\Delta_{n}(p / 2)}{n^{2 / p}}\left(\sum_{i=1}^{n}\left(\sigma_{i}^{2}\right)^{1 / 2}\right)^{2 / p}\right]^{n(n-1) / 2} \leqslant \frac{1}{n^{n(n-1) / p}}\left(\Delta_{n}(p / 2)\right)^{n(n-1) / 2},
$$

d'où

$$
\begin{aligned}
I_{n}=\int_{\Omega_{p}} \varphi(\sigma) d \sigma & \leqslant \operatorname{vol}\left(\Omega_{p}\right) \cdot \frac{1}{n^{n(n-1) / p}}\left(\Delta_{n}(p / 2)\right)^{n(n-1) / 2} \\
& \leqslant \operatorname{vol}\left(\Omega_{\infty}\right) \cdot n^{-n(n-1) / p}\left(\Delta_{n}(p / 2)\right)^{n(n-1) / 2} \\
& =\frac{1}{n !} n^{-m(n-1) / p}\left(\Delta_{n}(p / 2)\right)^{n(n-1) / 2},
\end{aligned}
$$

c'est-à-dire

$$
\limsup _{n \rightarrow \infty}\left(I_{n}^{p}\right)^{1 / n^{2}} \cdot n^{1 / p} \leqslant \sqrt{\Delta(p / 2)} .
$$

Inversement, il existe d'après le lemme 6 un $\tau^{*}=\left(\tau_{0}^{*}, \ldots, \tau_{n}^{*}\right)$ avec

$$
\tau_{0}^{*}=0, \quad\left\|\tau^{*}\right\|_{p / 2}=1, \quad \psi\left(\tau^{*}\right)=\left(\frac{\Delta_{n+1}(p / 2)}{(n+1)^{2 / p}}\right)^{n(n+1) / 2}
$$

et

$$
m=\inf \left(\tau_{k+1}^{*}-\tau_{k}^{*}\right) \geqslant\left(\frac{2}{n(n+1)}\right)^{1+2 / p} \geqslant\left(\frac{1}{n^{2}}\right)^{1+2 / p} \geqslant \frac{1}{n^{6}} .
$$

Posant alors $\sigma_{i}^{*}=\sqrt{\tau_{i}^{*}}, i=1,2, \ldots, n$, on a comme dans le théorème 3 , pour tout $\sigma$ tel que $\sigma_{i}^{*}-\delta m \leqslant \sigma_{i} \leqslant \sigma_{i}^{*}$,

$$
\varphi\left(\sigma_{i}\right) \geqslant \varphi\left(\sigma_{i}^{*}\right)(1-4 \delta)^{m(n-1) / 2}, \quad . \sigma \in \Omega_{p}
$$

et l'ensemble de ces $\sigma$ a une mesure égale à $(\delta m)^{n}$. Donc

$$
I_{n}^{p} \geqslant(\delta m)^{n} \varphi\left(\sigma^{*}\right)(1-4 \delta)^{n(n-1) / 2} .
$$

De plus

$$
\begin{aligned}
\varphi\left(\sigma^{*}\right) & =\prod_{1 \leqslant i<j \leqslant n}\left(\sigma_{j}^{* 2}-\sigma_{i}^{* 2}\right) \geqslant \prod_{i=1}^{n} \sigma_{i}^{* 2} \cdot \prod_{1 \leqslant i<j \leqslant n}\left(\sigma_{j}^{* 2}-\sigma_{i}^{* 2}\right) \\
& \geqslant \psi\left(\tau^{*}\right)=\left[\Delta_{n+1}(p / 2) \cdot(n+1)^{-2 / p}\right]^{n(n+1) / 2} .
\end{aligned}
$$

Donc

$$
\left(I_{n}^{p}\right)^{1 / n^{2}} \geqslant\left(\frac{\delta}{n^{6}}\right)^{1 / n}(1-4 \delta)^{(n-1) / 2 n}(n+1)^{\frac{-1}{p} \cdot \frac{n+1}{n}}\left(\Delta_{n+1}(p / 2)\right)^{\frac{n+1}{2 n}},
$$

c'est-à-dire, prenant $\delta=1 / n$

$$
\lim \inf \left(I_{n}^{p}\right)^{1 / n^{2}} \cdot n^{1 / p} \geqslant \sqrt{\Delta(p / 2)},
$$


d’où le résultat, dans le cas réel. Dans le cas complexe, les mêrnes inégalités donnent le résultat, compte tenu de ce que, sur $\Omega_{p}, \prod_{j=1}^{n} \sigma_{J} \leqslant 1$ et que

$$
\prod_{j=1}^{n} \sigma_{j}^{*} \cdot \prod_{j, k}\left(\sigma_{k}^{* 2}-\sigma_{j}^{* 2}\right) \geqslant \prod_{j=1}^{n} \sigma_{j}^{* 2} \cdot \prod_{j, k}\left(\sigma_{k}^{* 2}-\sigma_{j}^{* 2}\right)=\psi\left(\tau^{*}\right)
$$

donc que $|\widetilde{J}(1,1, \sigma)| \geqslant(1-\delta)^{n}(1-4 \delta)^{n(n-1)}\left[\psi\left(\tau^{*}\right)\right]^{2}$ sur un ensemble de mesure $\geqslant(\delta m)^{n}$.

Corollatre 8. Lorsque $n$ tend vers l'infini, on a

$$
\begin{aligned}
& \left(\operatorname{vol}\left(B_{n}^{p}\right)\right)^{1 / n^{2}} \sim \frac{\sqrt{2 \pi e^{3 / 2} \cdot \Delta(p / 2)}}{n^{1 / 2+1 / p}}, \\
& \left(\operatorname{vol}\left(\tilde{B}_{n}^{p}\right)\right)^{1 / 2 n^{2}} \sim \frac{\sqrt{\pi e^{3 / 2} \cdot \Delta(p / 2)}}{n^{1 / 2+1 / p}} .
\end{aligned}
$$

En particulier $\Delta(1)=e^{-1 / 2}$.

Ceci résulte immédiatement des théorèmes 1,2 et 7 . Dans le cas $p=2$, $B_{n}^{2}$ est la boule unité euclidienne de $\boldsymbol{R}^{\boldsymbol{n}^{2}}$. Donc

$$
\left(\operatorname{vol}\left(B_{n}^{2}\right)\right)^{1 / n^{2}}=\left(v_{n^{2}}\right)^{1 / n^{2}}=\frac{\sqrt{\pi}}{\left(\Gamma\left(1+n^{2} / 2\right)\right)^{1 / n^{2}}} \sim \frac{\sqrt{\pi}}{\sqrt{\frac{n^{2}}{2}} e^{-1 / 2}}=\frac{\sqrt{2 \pi} e}{n},
$$

d'où

$$
\frac{\sqrt{2 \pi e^{3 / 2} \cdot \Delta(1)}}{n}=\frac{\sqrt{2 \pi} e}{n},
$$

c'est-à-dire $\Delta(1)=e^{-1 / 2}$

THÉORÈME 9. Le quotient volumique (= volume ratio) de la boule nucléaire $B_{n}^{1} a$ une limite inférieure à $2 \cdot e^{-1 / 4}$ quand $n$ tend vers l'infini.

La boule $B_{n}^{1}$ est le polaire de la boule $B_{n}^{(x)}$. L'inégalité de BlaschkeSantaló ([1], [3]) entraîne donc

$$
\operatorname{vol}\left(B_{n}^{1}\right) \cdot \operatorname{vol}\left(B_{n}^{x \times}\right) \leqslant\left(v_{n^{2}}\right)^{2}
$$

et donc $\left[\operatorname{vol}\left(B_{n}^{1}\right)\right]^{1 / n^{2}} \cdot\left[\operatorname{vol}\left(B_{n}^{(x)}\right)\right]^{1 / n^{2}} \leqslant\left[\operatorname{vol}\left(B_{n}^{2}\right)\right]^{2 / n^{2}}$, c'est-ì-dire $\Delta(1 / 2) \times$ $\times \Delta(\infty) \leqslant(\Delta(1))^{2}=e^{-1}$, ou encore, puisque $\Delta(\infty)=1 / 4, \Delta(1 / 2) \leqslant 4 e^{-1}$.

L'unicité de l'ellipsoïde de John de $B_{n}^{1}$ montre qu'il est invariant par $\mathrm{SO}(n)$, donc que cet ellipsoïde est un homothétique de la boule $B_{n}^{2}$; le rapport d'homothétie est alors évidemment $n^{-1 / 2}$, d'où

$$
\operatorname{vr}\left(B_{n}^{1}\right)=\frac{\left(\operatorname{vol}\left(B_{n}^{1}\right)\right)^{1 / n^{2}}}{n^{-1 / 2}\left(\operatorname{vol}\left(B_{n}^{2}\right)\right)^{1 / n^{2}}} \rightarrow \sqrt{\frac{\Delta(1 / 2)}{\Delta(1)}} \leqslant 2 e^{-1 / 4}<2 .
$$

Ceci améliore, au moins pour les grandes valeurs de $n$, un résultat de Szarek et Tomczak-Jaegermann qui majorent vr $\left(B_{n}^{1}\right)$ par 32000 . En fait, en raffinant les majorations faites ici, on obtient avec des calculs fastidieux

$$
\sup _{n} \operatorname{vr}\left(B_{n}^{1}\right)<5 \text {. }
$$

\section{Bibliographie}

[1] W. Blaschke, Uther affine Geometrie VII. Neue Exiremeigenschaften von Ellipse und Ellipsoid, Sitz. Ber. Akad. Wiss. Leipz. Math. Nat. K1. 69 (1917), 306-318.

[2] E. Hille, Analytic functions theory II, Chelsea Publishing Company, New York.

[3] L. A. Santaló, Un invariante afin para los cuerpos convexos del espacio de $n$ dimensiones, Portugal. Math. 8 (1949), 155-161.

[4] S. J. Szarek and N. Tomczak-Jaegermann, On nearly euclidean decomposition for some classes of Banach spaces, Compositio Math. 40 (1980), 367-385.

ÉQUIPE D'ANALYSE, UNIVERSITÉ PARIS VI 4. Place Jussieu, 75230-Paris Cedex 05 4, Place Jussieu, 75230 